\title{
STELLAR FLARES: OBSERVATIONS AND THEORY
}

\author{
Suzanne L. Ilawley \\ Astronomy Department, University of Texas at Austin
}

\begin{abstract}
Photometric and spectroscopic observations of a very large flare on AD Leo are presented. A self consistent model of a flare corona, transition region and chromosphere is developed; in particular the chromospheric temperature distributions resulting from X-ray and EUV irradiation by coronae of various temperatures are determined. The predicted line fluxes in $\mathrm{H} \gamma$ are compared to the observed line fluxes to find the coronal temperature as a function of time during the flare. This run of temperature with time is then compared with the predictions of an independent theoretical flare model based on a dynamic scaling law (see paper by Fisher and Hawley, these proceedings).
\end{abstract}

\section{OBSERVATIONS}

- 4.5 magnitude (in U) flare on AD Leo observed on $12 \Lambda_{p}$ rij 1985 U'T.

- Optical spectroscopy, McDonald Olsservatory, $2.1 \mathrm{~m}$ telescope, cassegrain spectrograph, CCD detector. Observations extended for more than 5 hours and are spectrophotometric to $\pm 10 \%$. Spectral resolution was $3.5 \AA$; spectra ware taken every 1 to 3 minutes.

- Optical photometry, McDonald Observatory, $0.9 \mathrm{~m}$ telescope, eight filters (U, $B, V, R$, Ho narrow and wide, $\mathrm{H} \beta$ narrow and wide). Observations made by B.R. Pettersen.

- IUE short wavelength (SW/P) spectrum includes the first 15 minutes of the flare. IUP long wavelength (LWP) spectrit, time resulution of 3 to 8 minules obtained by moving star to five positions in the aperture during each exposure. Coverage begins 20 minntes after $U$ band flares. Saturated regions marked in red. Observations made by B.N. Andersen.

- Quiescent spectra for comparison. Note greatly mag!ified flux scales.

- Continuum fluxes per $\AA$ are presented in Figure 3. The flux in $U$ and $V$ was obtained using photometric calibration fron: stindard stars. Fluxes at 2800 and $2100 \AA$ were obtained from the IUE spectra. The $2800 \AA$ flux is a lower limit.

- Equivalent width indices for $\mathrm{II} \alpha$ and $1 \mathrm{l} / 3$ were found by dividing the counts in the narrow filter by the counts in the wide filer after sky subtraction. An equivalent Il $\gamma$ index was computed from the optical spectra and is included for comparison in Figure 4.

- Line fluxes for $\mathrm{H}_{\gamma}, \mathrm{H} \delta, \mathrm{Ca}$ II $\mathrm{K}$ and $\mathrm{Mg} / 1 h+k$ are presented in Figure 5. Nole that $\mathrm{Ca}$ II $\mathrm{K}$ las a broad flat peak compared to the Balmer lines. 'The first five $M_{g}$ II points are lover limits. The If ${ }_{\gamma}$ flux is ssed as an example in the modeling 


\section{THEORY}

'The goial is to develop a simple but self consistent model of the corona, transition refion and chromosphere that includes all the inportint heating and cooling processes that result after an initial burst of encrgy is deposited in the corona.

- Coronial scaling liw (Fisher and Jawley, this meeting) used to determine coronal pressure, columu depth and temperature structure for various values of comial apex temperature.

- Lrom temperature structure, emergent downward directed X-ray flux can be computed.

- Column depth determines where transition region begins; T.R. structure compulced l,y equating conductive flux with optically thin metal losses.

- (hrouospheric structure determined using the Scharmer-Carlsson NLTE radiative triusfer method to solve IISE, statistical equilibrium and radiative

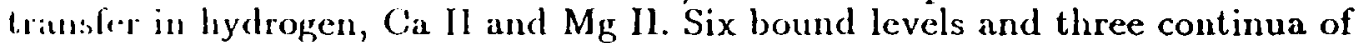
hydrogren are includid explicitly. The temperature structure is then adjusted until the couputed $X$-ray lieating from the $X$-ray flux is balanced by the radiative cooling at cach depth level in the atmosphere. Resultant atmospheres for coronial apex temperatures of $3 \times 10^{8} \mathrm{~K}$ (the quiescent model), $8 \times 10^{6} \mathrm{~K}$, $10 \times 10^{19} \mathrm{~K}, 15 \times 10^{\circ} \mathrm{K}$, and $20 \times 10^{\circ} \mathrm{K}$ are shown in Figure 6. Significant temperature minimum and photospheric heating occurs as a result of back-, warming in the optical and ultraviolet continua. The X-ray heating in these aluosipheres is shown in Pigure 7.

- Lime and continumu fluxes computed fromthe nodels can be plotted as a function of coronal apex temperature. $\mathrm{H}_{\gamma}$ is shown in Figure 8 . When combined with the olserved line fluxes (Figure 5), the olsservationally predicted apex f(m)rerature is ubtained as a function of time. This is shows for the AD leo flare in lighure 9.

- (Our Hare loop evolution model (Fisher and Hawley, this meeting) predicts the tinke cvolution of the apex temperature for given values of loop length, heating tinke and volumetric heating rate in the corona. We choose values of $10^{10}$ (111, 1000 seconds (the observed Bahmer line rise tinte) and $1 \mathrm{erg} \mathrm{sec}^{-1} \mathrm{~cm}^{-2}$, resulting in a total energy deposition of $10^{13} \times$ (llare area) sigs. Approximately ten precent of the stellar surface must be firring at this rate to match the observed ratiative flare energy.

- Whe loop mudel evolution is shown in Figure 10, and is to be compared with Figure 9. It appears that the model is able to reproduce some important features of the observations, such as the shape of the rise phase and initial decay. Continued heating would be necessary to match the long decay phase. The application of this simple but self-consistent model indicates that X-ray heating probahly plays an important role in stellar flares, and must be included in any detailed inodel. 
Figure 3

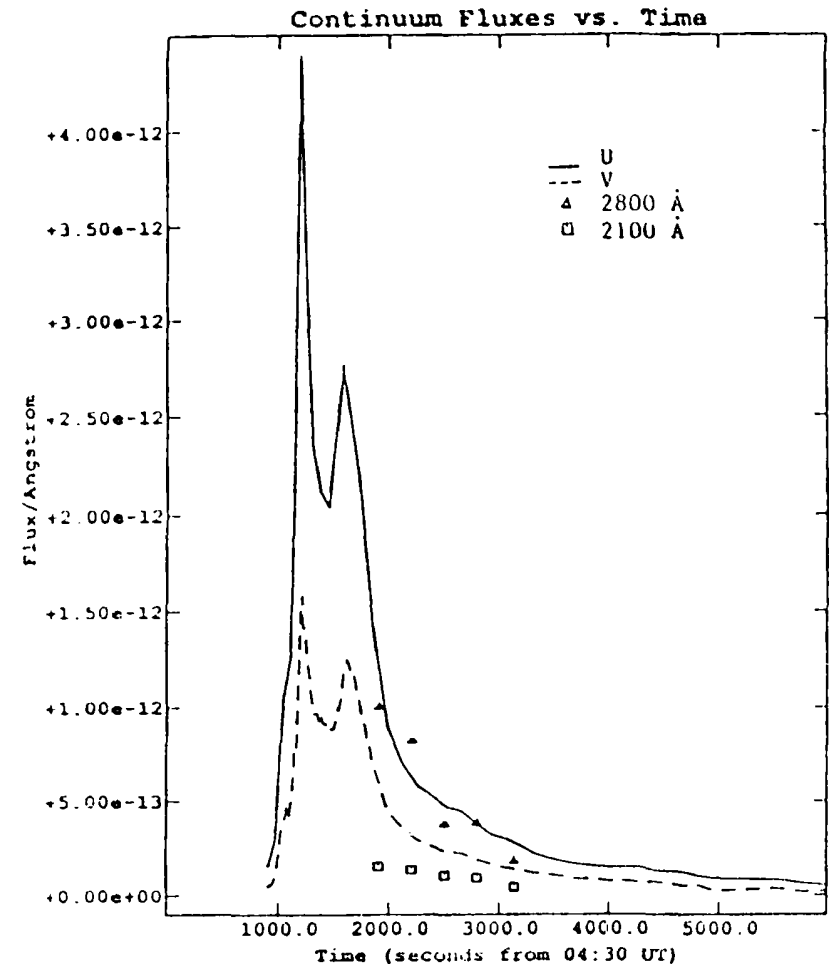

Figure 6

Chroundipheric Temperacure Dlelributions fur Various Coronal Apex lempicilures

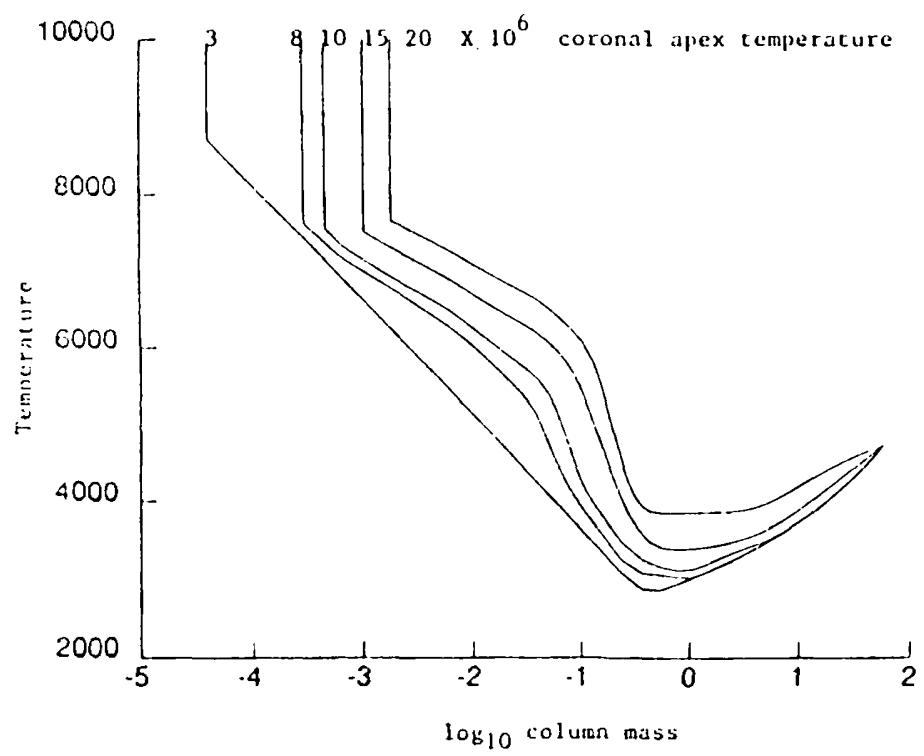

Finute 5

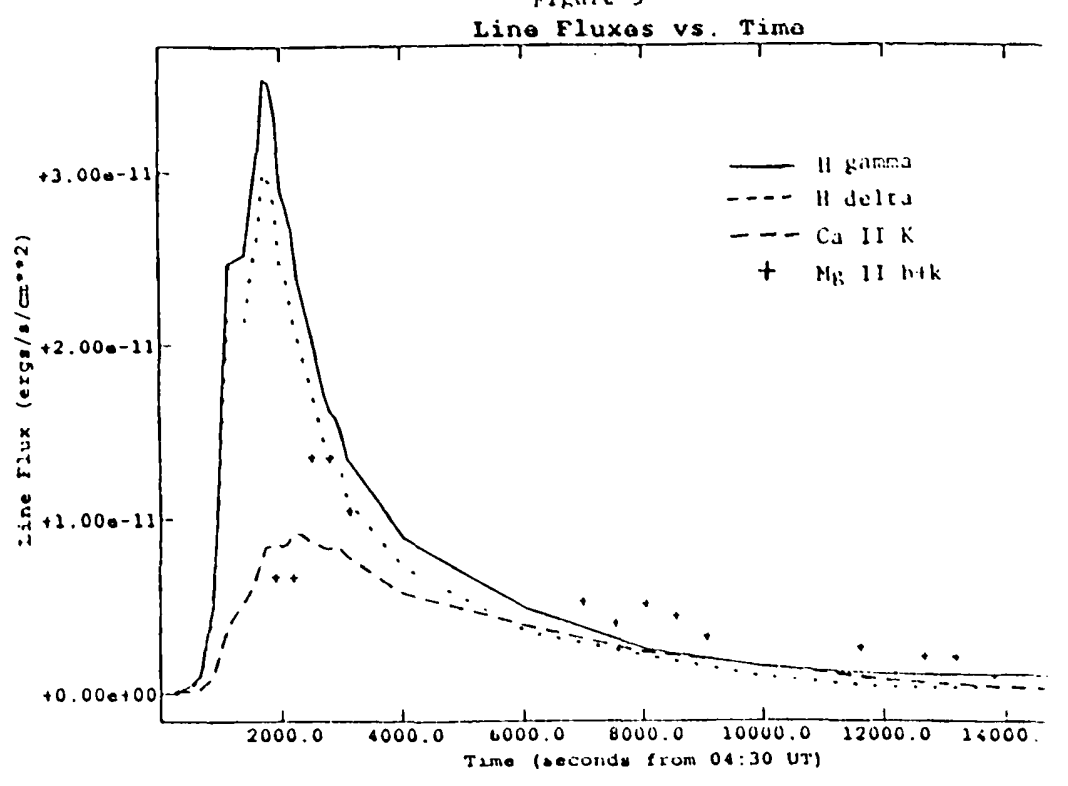

Fipute?

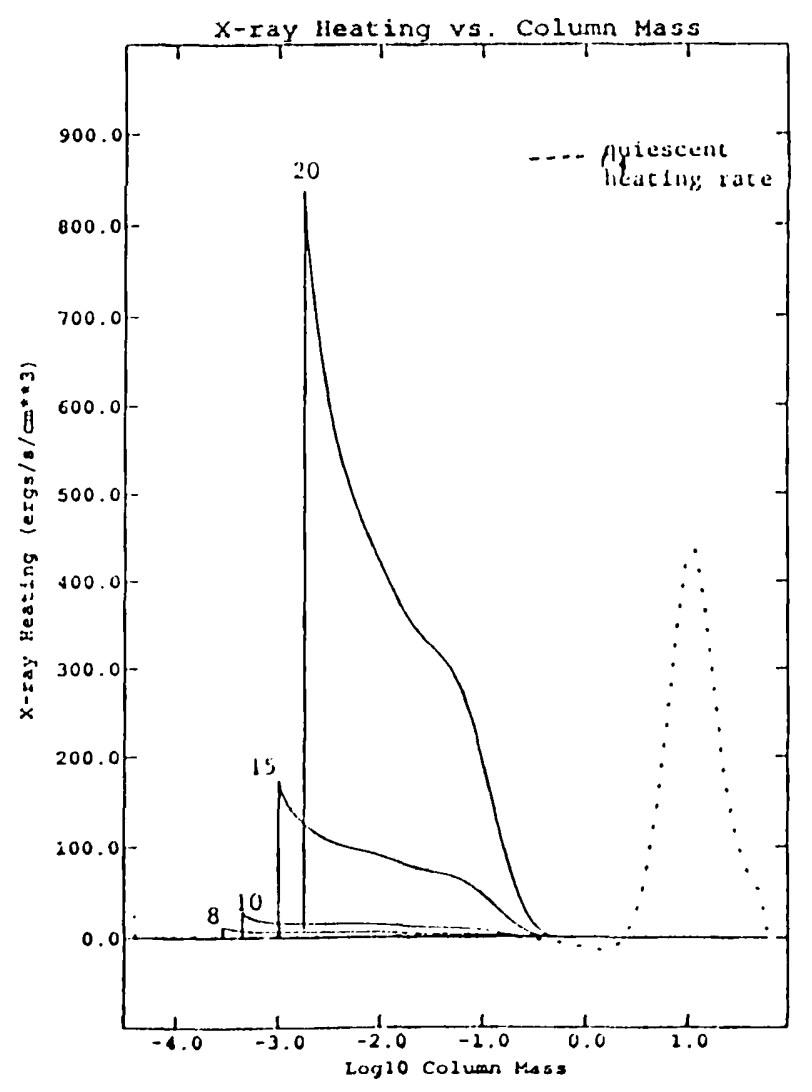


biure to

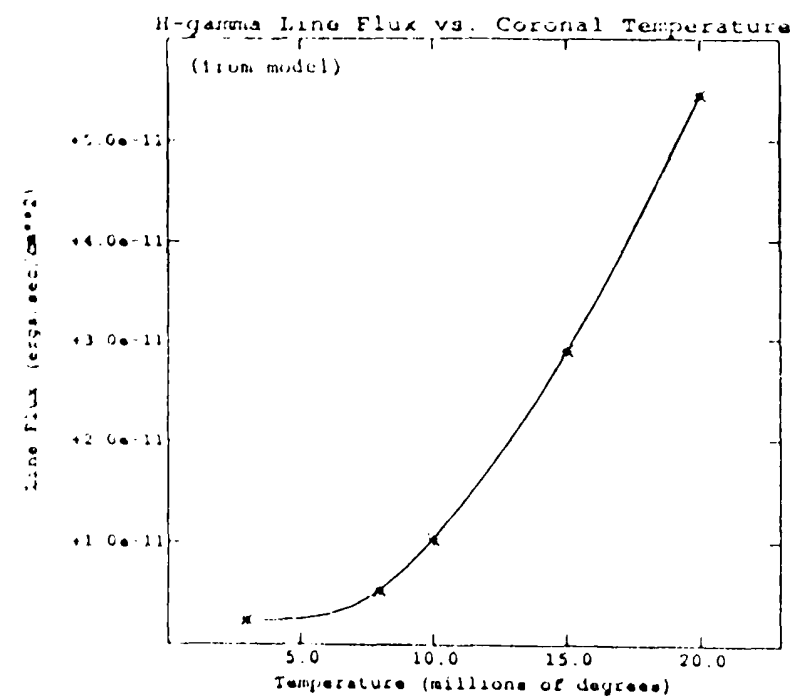

Finde: 9

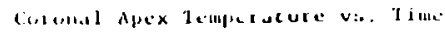

(1, an H kistum ol setervat forts)

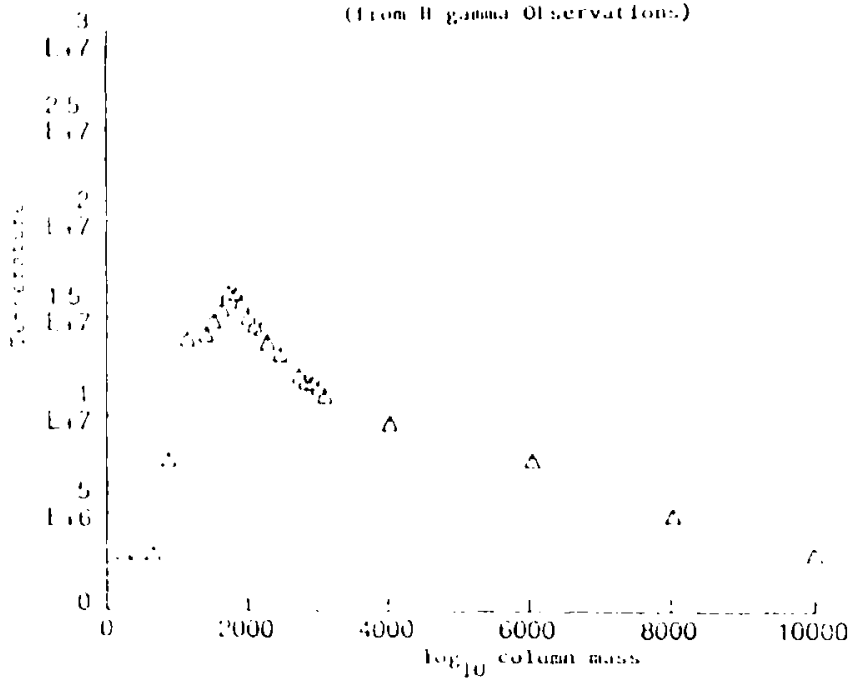

Heute 10

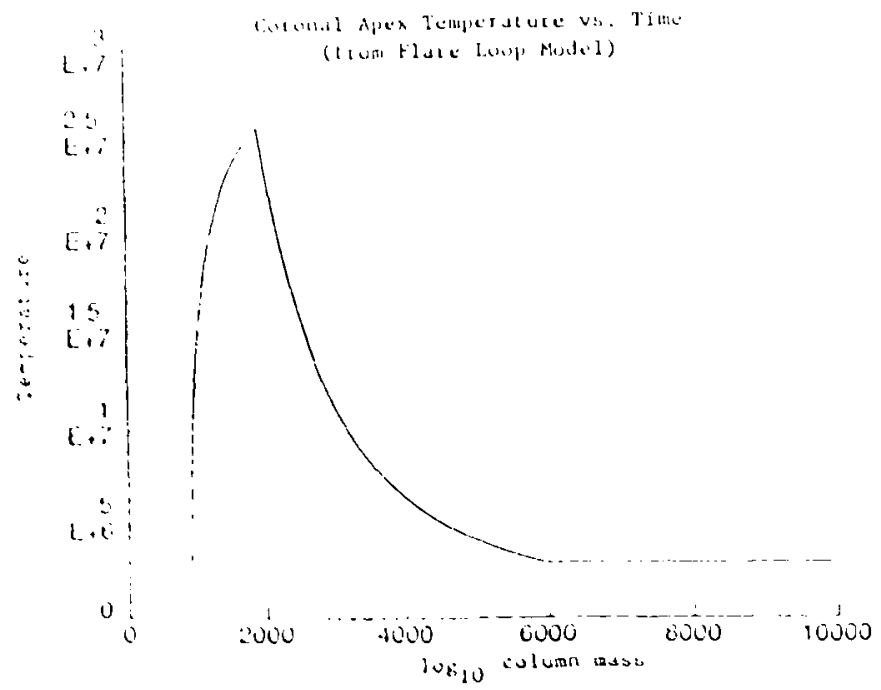

